PHYSICAL REVIEW D 93, 109901(E) (2016)

\title{
Erratum: Teleparallel quintessence with a nonminimal coupling to a boundary term [Phys. Rev. D 92, 084034 (2015)]
}

Sebastian Bahamonde and Matthew Wright (Received 25 April 2016; published 9 May 2016)

DOI: 10.1103/PhysRevD.93.109901

We have observed an error in one of our cosmological field equations in Sec. IV. The expression for pressure of the scalar field should have read

$p_{\phi}=\frac{1}{2}(1-4 \chi) \dot{\phi}^{2}-V(\phi)+2 H \phi \dot{\phi}(2 \xi+3 \chi)+2 \phi^{2} \dot{H}\left(\xi+6 \chi^{2}\right)+2 \chi \phi V^{\prime}(\phi)+3 H^{2} \phi^{2}\left(\xi+8 \chi^{2}\right)+12 \chi H^{2} \phi^{2}(\xi+\chi)$.

The last term of this expression is the additional term missed in the original paper. This has little effect on the main results of the paper. The system still generically evolves to a late time accelerating attractor solution, and a dynamical crossing of the phantom barrier is still possible. This mistake was carried throughout the paper though, and below are the corrected equations.

Firstly, the claim in the last paragraphs of Sec. IV that the matter conservation law does not hold is no longer true. In fact now

$$
\dot{\rho}+3 H(\rho+p)=0
$$

and also

$$
\dot{\rho_{\phi}}+3 H\left(\rho_{\phi}+p_{\phi}\right)=0 \text {. }
$$

The dynamical system is modified to become

$$
\begin{gathered}
x^{\prime}=-\frac{\sqrt{6} y^{2} \lambda(x z-2)+3(2 x+z)\left(x^{2}(4 \chi+w-1)+x(w-1) z+y^{2}(w+1)-w+1\right)}{\left(z^{2}+4\right)}, \\
y^{\prime}=-\frac{y\left(\sqrt{6} \lambda\left(x\left(z^{2}+4\right)+2 y^{2} z\right)+4\left(3 x^{2}(w-1)+3 x(w-1) z+3 y^{2}(w+1)\right)-6\left(2 w+2+z^{2}\right)\right)}{2\left(z^{2}+4\right)}, \\
z^{\prime}=12 \chi x .
\end{gathered}
$$

Now the effective EoS becomes

$$
w_{\mathrm{eff}}=-\frac{12 x^{2}(4 \chi+w-1)+12(w-1) x z+2 y^{2}(\sqrt{6} \lambda z+6 w+6)-3\left(z^{2}+4 w\right)}{3\left(z^{2}+4\right)} .
$$

Only the critical point $D$ is modified, now being

\begin{tabular}{lllll}
\hline Point & $x$ & $y$ & $z$ & Existence \\
\hline$D$ & 0 & 1 & $\sqrt{\frac{2}{3} \lambda}$ & $\forall \lambda, \chi$ \\
\hline
\end{tabular}


Its eigenvalues and stability become

\begin{tabular}{|c|c|c|c|c|}
\hline Point & $w_{\text {eff }}$ & Acceleration & Eigenvalues & Stability \\
\hline$D$ & -1 & Yes & $\begin{array}{c}-3, \frac{3}{2}\left(-1+\frac{\sqrt{\lambda^{2}-48 \chi+6}}{\sqrt{\lambda^{2}+6}}\right), \\
\frac{3}{2}\left(-1-\frac{\sqrt{\lambda^{2}-48 \chi+6}}{\sqrt{\lambda^{2}+6}}\right)\end{array}$ & $\begin{array}{c}\text { Stable spiral: } 48 \chi>\lambda^{2}+6 \\
\text { Stable point: } 0<48 \chi<\lambda^{2}+6 \\
\text { Saddle point: } \chi<0\end{array}$ \\
\hline
\end{tabular}

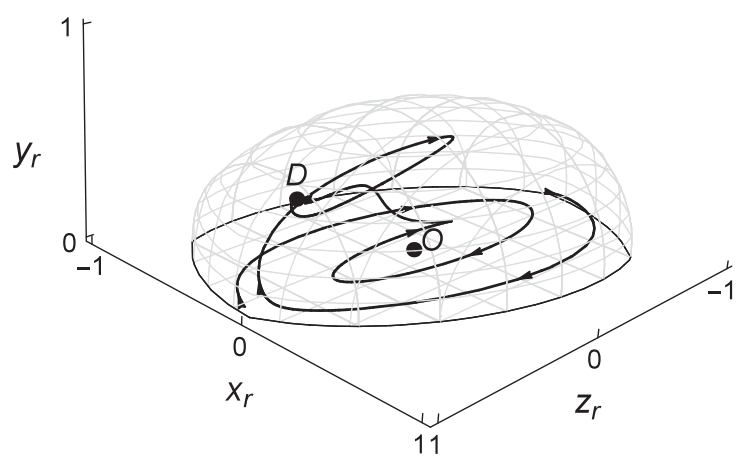

FIG. 1. Phase space showing trajectories of the dynamical system (4)-(6) in Poincaré variables when $\chi=1, \lambda=2$, and $w=0$. Point $D$ is the global attractor.

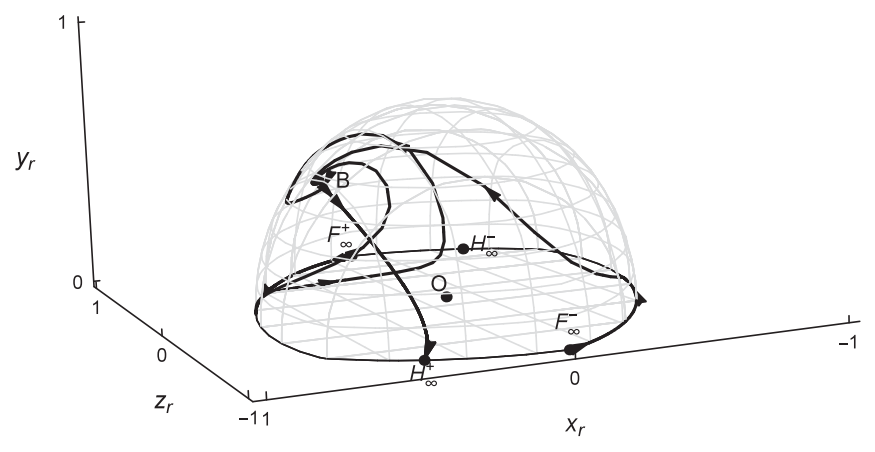

FIG. 2. Phase space showing trajectories of the dynamical system (4)-(6) when $\chi=-10^{-3}, \lambda=2$, and $w=0$. Trajectories end at unphysical critical points lying at infinity.

In addition, Eq. (62) is modified to become

$$
\theta^{\prime}=24 \chi \cos \theta \cot \theta(\sin \theta+\cos \theta)-\frac{3}{2}(2 \cot \theta+1)(\sin (2 \theta)+\cos (2 \theta)+1) .
$$

However, it is still true that none of the critical points lying at infinity are physical; they all have an undefined dark energy density parameter.

A couple of the figures are modified slightly; however, their broad behavior does not change. 